\title{
Hearing with Bone-anchored Hearing Aid (BAHA)
}

\section{${ }^{1}$ Gauri Mankekar, ${ }^{2}$ Payal Bhattacharya Chitranshi, ${ }^{3} \mathrm{MV}$ Kirtane}

${ }^{1}$ ENT Consultant, PD Hinduja Hospital, Mahim, Mumbai, Maharashtra, India

${ }^{2}$ Clinical Assistant, PD Hinduja Hospital, Mahim, Mumbai, Maharashtra, India

${ }^{3}$ Professor Emeritus, GS Medical College and KEM Hospital, Consultant ENT Surgeon, PD Hinduja Hospital, Saifee Hospital and Breach Candy Hospital, Mumbai, Maharashtra, India

Correspondence: Gauri Mankekar, ENT Consultant, PD Hinduja Hospital, Mahim, Mumbai-16, Maharashtra, India

\section{Abstract}

BAHA is a surgically implantable system for treatment of hearing loss in patients wherein conventional hearing aids are unable to provide benefit. e.g. patients with microtia, chronic ear discharge or single sided hearing loss.

Keywords: Bone anchored hearing aid, osseointegration, sound processor, abutment, fixture.

\section{INTRODUCTION}

BAHA is a surgically implantable system for treatment of hearing loss. The system is surgically implanted and allows sound to be conducted through the bone rather than via the middle ear by a process known as direct bone conduction.

Conventional hearing aids aim to overcome hearing loss by amplifying the incoming signal and sending the amplified sound via air conduction through the cochlea. Cochlear implants on the other hand, are suitable for individuals with bilateral severe to profound hearing loss, where the extent of destruction of the cochlear hair cells is so great that hearing aids are unable to provide benefit. BAHA provides an ideal choice for those with conductive, mixed or a single sided sensorineural deafness through direct bone conduction. The prerequisite for a BAHA is a functioning cochlea.

\section{HISTORY}

In the early 1960's Professor Branemark, a dentist, discovered that titanium is accepted by the human body and bonds with the surrounding bone thus forming a permanent structure. He termed this process "osseointegration” (Fig. 1). Osseointegrated implants were first introduced in dentistry in Sweden during the 1970s. Boneanchored implants were first described in, 1977 by Tjellstrom to treat patients with a conductive or mixed hearing loss (HL) who could not use a conventional air conduction hearing aid to amplify and improve their hearing. ${ }^{1}$

The BAHA has its origin with dental implant technology, in which titanium screws are placed within the bone of the maxilla or mandible, where they are osseointegrated,

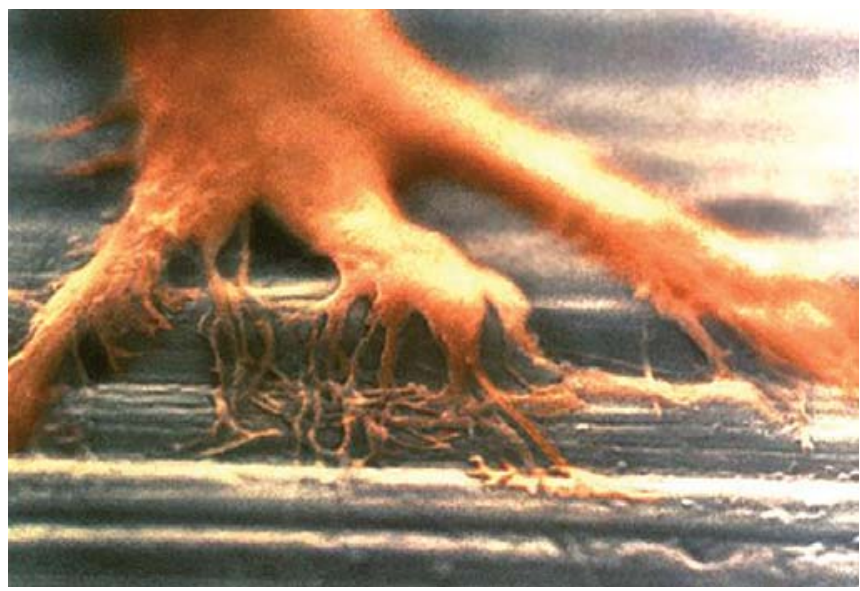

Fig. 1: Osseointegration (Courtesy: Cochlear Ltd., Australia)

becoming rigidly and permanently fixed to the bone. These screws then serve as the basis for prosthetic crowns that can be applied after osseointegration. In the case of BAHA, the principle is identical. The fixture consists of a titanium screw, literally, that is surgically implanted into the bone directly behind the ear. Attached to the fixture, but not actually integrated into the bone, is also a titanium component, the abutment, which is held onto the fixture by means of a very small central screw.

The use of osseointegrated implants for bone-anchored hearing aid (BAHA) retention is now a established practice since the device became commercially available in 1987. The surgical implantation technique has gradually been simplified. The original 2-stage surgical procedure was reduced to a 1-stage procedure in 1989 and has remained the surgical standard in adults since then. In the early 90s, 
a simplified surgical technique was developed step-by-step in Nijmegen. ${ }^{2}$

BAHA was cleared by the US FDA in 1996 as a treatment for conductive and mixed hearing losses in the United States. In 2002, the FDA approved its use for the treatment of unilateral sensorineural hearing loss. The acronym BAHA is a trademark. In 2005, Entific sold the rights of BAHA to Cochlear. Cochlear realized that many insurance companies were having trouble distinguishing the BAHA as a sound processor and not a hearing aid, which makes a huge difference for insurance coverage. They removed the acronym and have since called it strictly the BAHA.

\section{COMPONENTS OF BAHA (FIG. 2)}

BAHA has 3 components (Fig. 2). They are:

1. The sound processor: A detachable electronic hearing aid with a snap-fit coupling to the abutment. The user takes the sound processor on and off as required, for example for hair washing or swimming.

2. The abutment: A socket attached by an internal screw to the fixture. The abutment penetrates the surface of the scalp and is shaped to hold the snap-fit coupling of the sound processor. The abutment can be unscrewed from the fixture for maintenance or replacement by the specialist audiologist.

3. The fixture (or implant): A small titanium screw, four millimeters long, implanted into the bone behind the ear. The fixture is permanent, it is not adjusted or removed. The metal becomes firmly anchored to living bone by the process of osseointegration.

The system works by taking the sound from the outside and transmitting it to the inner ear through the bone. This bypasses the ear canal and the middle ear. The titanium implant is placed during a minor surgical procedure and

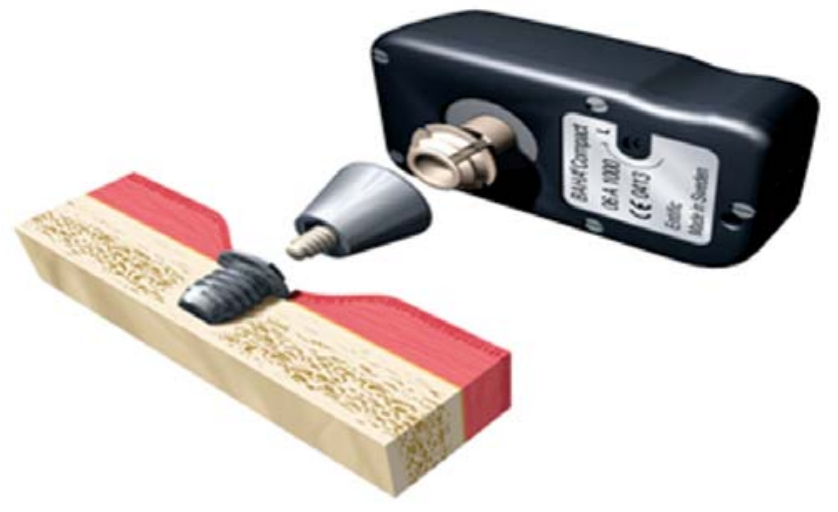

Fig. 2: Components of BAHA (Courtesy: Cochlear Ltd., Australia) over time integrates with the bone behind the ear. The hearing device transmits sound vibrations through the titanium implant to the skull and the inner ear, where the hearing takes place.

\section{INDICATIONS FOR BAHA}

The BAHA is used to rehabilitate people with conductive and mixed hearing impairment. This includes people with chronic infection of the ear canal, people with absence of or a very narrow ear canal as a result of a congenital ear malformation, infection, or surgery, and people with a single sided hearing loss as a result of surgery for a vestibular schwannoma.

1. Conductive hearing loss: BAHA is frequently the ideal choice for conductive hearing loss. This is because conductive loss is often concomitant with various outer and middle ear abnormalities (e.g. atresia) or middle ear pathologies like continuously draining ear that prelude the wearing of conventional hearing aids. With BAHA the conductive element of the hearing loss is bypassed by sending sound vibrations directly from the BAHA through the skull to the cochlea. BAHA often provides benefits over conventional hearing aids in large conductive losses due to their technical limitations of insufficient gain, saturation, feedback and necessity of wearing a tight ear mould.

2. Mixed hearing loss: BAHA provides two-fold solution to all such patients. First, it closes the air-bone gap by bypassing the conductive element. Second, it compensates for the remaining degree of sensorineural hearing loss. The overall amplification required for people with a mixed hearing loss is less with BAHA than conventional hearing aids and it is recommended when conductive component of the mixed hearing loss is greater than 30 $\mathrm{dB} .^{3,4}$

3. Single-sided sensorineural deafness (SSD): SSD causes significant communication difficulties for the patient due to inability to localize sound, more so when noise is directed towards the good ear and the talker is near the deaf ear. BAHA is worn on the deaf side and transfers the signal directly across the skull via bone conduction thus eliminating the head shadow effect. But these patients must have normal hearing in the contra-lateral ear (20 dB HL air conduction pure tone averages). BAHA provides better directional 360 degree hearing in these patients. Studies have shown that patients with BAHA have better speech understanding than those with Contralateral Routing of Signal (CROS) hearing aids. ${ }^{5}$ 


\section{HOW DOES THE BAHA WORK?}

The principle of bone conduction for hearing forms the basis for both simple and complex tests of hearing function (ranging from tuning fork testing at the bedside to extended frequency bone conduction threshold audiometry). Sound, which is a form of vibrational energy, requires a medium in which to travel. Typically, we consider this medium to be the environmental air. Yet the moment that sound reaches the eardrum, it no longer travels through the air. Rather, through an elegant process of impedance matching and energy amplification, sound waves ultimately produce a wave of energy that vibrates fluids within the inner ear, leading to the production of neural impulses that travel down the cochlear nerve. Because the inner ear is encased in bone (the temporal bone of the skull), the normal pathway of hearing from eardrum to inner ear is not the only means of provoking vibration within its fluids. By placing a sound source directly on the bones of the skull, sound will travel through the skull using the bone itself as a medium, where it in turn causes fluids of the inner ear to vibrate, leading to production of neural impulses and sound perception. This mechanism forms the basis by which traditional bone conduction hearing aids work.

BAHA technology addresses these issues precisely. The titanium implant is placed during a short surgical procedure and over time osseointegrates with the skull bone. For hearing, the sound processor transmits sound vibrations through the external abutment to the titanium implant. The vibrating implant sets up vibrations within the skull and inner ear that finally stimulate the nerve fibers of the inner ear, allowing hearing.

\section{PREOPERATIVE ASSESSMENT}

\section{Audiological Assessment}

- Pure tone audiometry (air conduction and bone conduction): Depending on the results of the bone conduction audiogram, it is decided whether a head mounted sound processor, or the more powerful bodyworn sound processor would be suitable.

- A headband test: The candidate gets the opportunity to "try out" a BAHA on a headband. The sound quality from the headband is not nearly as good as the real thing, because the sound is muffled by having to pass through the soft tissues of the scalp. If the candidate can hear reasonably well with the headband BAHA, then he will hear better with the implant proper.

\section{Surgical Assessment}

- BAHA is commonly used to treat conductive hearing loss due to chronic ear infections. It can also be used to treat single sided sensorineural deafness, where one cochlea (inner ear) is no longer working. Before recommending BAHA as a treatment, a specialist assessment is needed to confirm the diagnosis. Ear microscopy with suction of the ear may be needed.

- HRCT temporal bone axial (Fig. 3) and coronal cuts with simple cursor markings to determine the thickness of the bone in front of and behind the temporoparietal suture line. The implant fixture is sited just behind the suture line. If the cortical bone thickness is less than $3 \mathrm{~mm}$ then a two stage BAHA surgery is recommended but if the bone thickness is more than $3 \mathrm{~mm}$, then a single stage (fast) surgery can be performed.

- It is important to know the patients expectations from BAHA and to decide whether the BAHA is likely to fulfil those expectations since having any form of implant surgery is an important decision with a lifelong commitment.

\section{SURGERY}

In recent years, improvements in the surgical procedures have resulted in most patients being able to have a single stage (fast) procedure carried out under local or a short

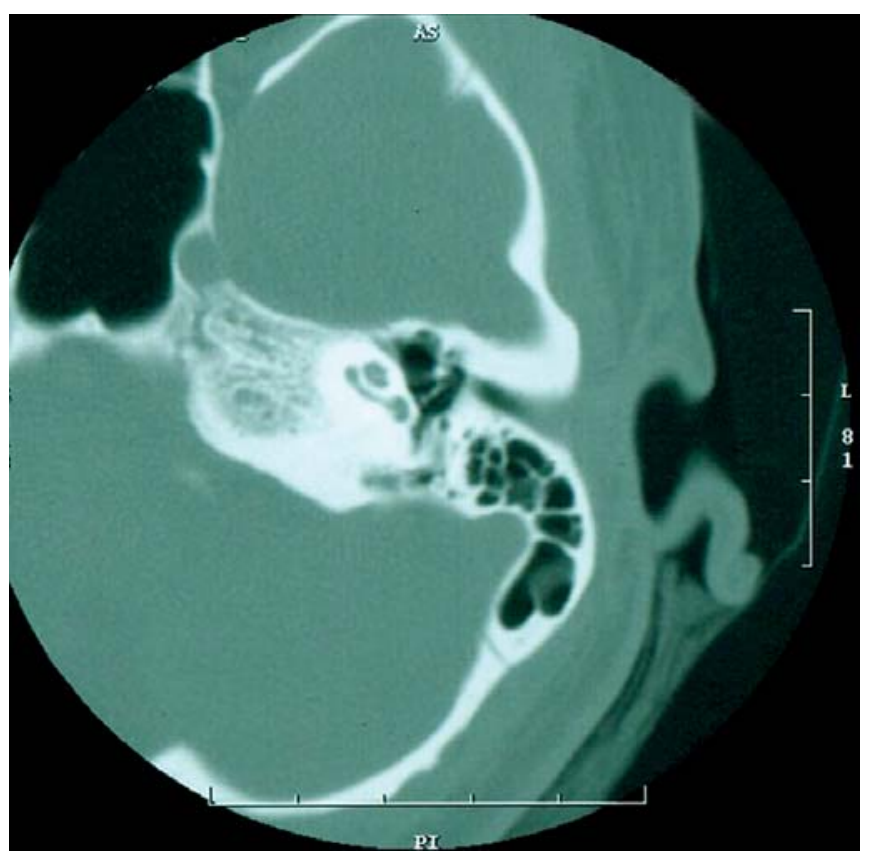

Fig. 3: HRCT temporal bone to confirm thickness of bone around the temporoparietal suture 
general anesthesia. Most patients are able to leave hospital within a few hours, or the day after their procedure and many patients do not even require analgesia in the following days.

In many cases, the surgeon will implant a second "sleeper" receptacle for use should the initial receptor fail or be damaged. ${ }^{6}$

A double stage procedure is sometimes carried out for small children/babies and for patients with a previously irradiated temporal bone wherein the bone thickness is inadequate for the fixture to be implanted. The first stage involves marking the incision (Fig. 4) — the canthomeatal line, mastoid tip are identified. The site is selected 5 to 5.5 $\mathrm{cm}$ from the external auditory meatus so that any planned pinna reconstruction (in cases with microtia/anotia) or the pinna will not influence the BAHA processor. Using the

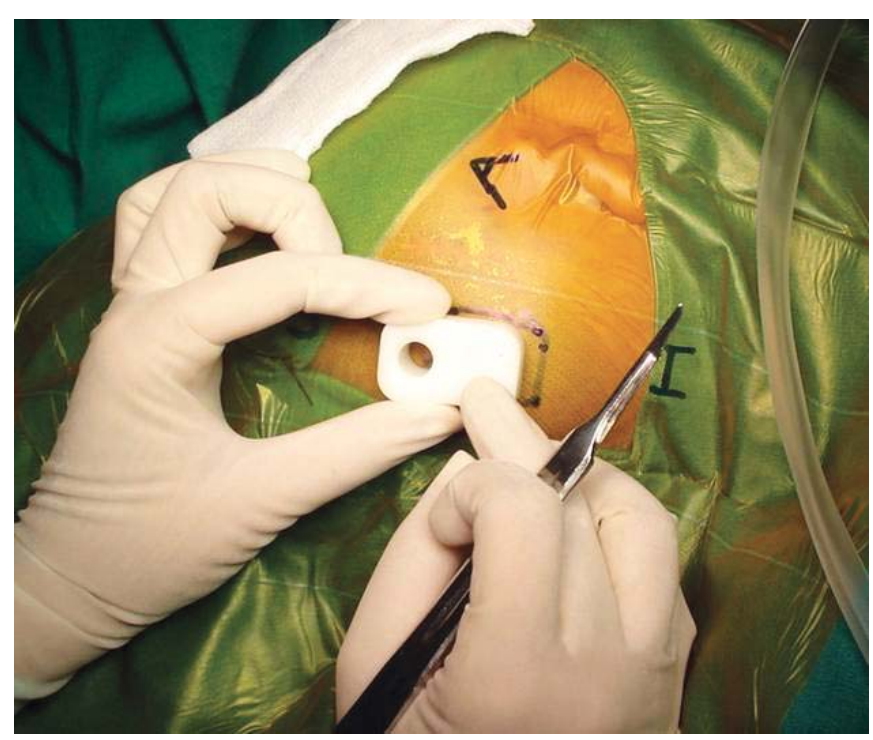

Fig. 4: Marking the site of incision

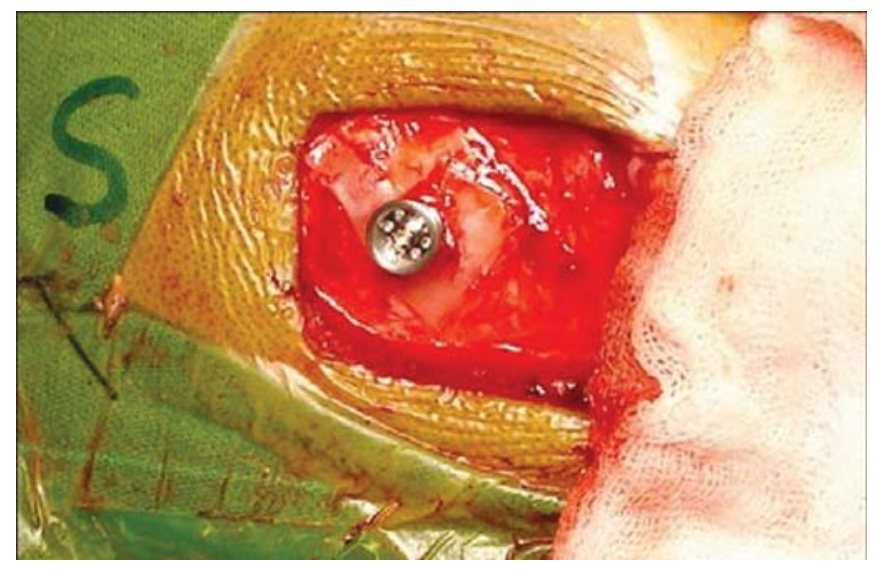

Fig. 5: Fixture abutment insertion
BAHA marker, the site is selected and marked with ink. Using methylene blue and a needle, the marking is extended through the skin and subcutaneous tissue to the mastoid bone. Then a small 1 by $1 \mathrm{~cm}$ section of skin behind the ear is peeled back followed by drilling one or more holes for the fixture. The minimum recommended fixture depth is 3 $\mathrm{mm}^{7}$ (Fig. 5).

Throughout the surgical procedure, the surgeon must keep thermal and mechanical trauma to a minimum to ensure adequate osseointegration. The skin is then grafted back and the child waits for the skull to continue to thicken and for osseointegration to occur properly. After osseointegration (a minimum of 3 to 6 month period), the second stage is performed. The second stage is another surgery in which the "post" is attached, and the skin is grafted around the "post". In the second stage, a skin flap is created using a Dermatome (Fig. 6) designed specifically for BAHA surgery. The dermatome creates a skin flap with a thickness of $0.6 \mathrm{~mm}$. At this thickness, the surgeon is ensured of a depilated flap of sufficient depth for proper wound healing. Then the soft tissue around the fixture implant is reduced up to a circumference of at least $1.5 \mathrm{~cm}$ with more soft tissue being removed superiorly. The removal of surrounding subcutaneous tissue is a crucial step in BAHA because shorter lengths have a much greater chance for failure. ${ }^{8}$ The titanium fixture should never come in contact with anything other than implant. Removal of soft tissue helps to prevent postoperative prolapse of soft tissue onto the abutment with subsequent granulation tissue around the implant. Therefore the tissue reduction should result in an area of very thin, hairless skin around all around the implant. Finally, prior to securing the skin flap, the periosteum under the skin flap should be thinned to one layer thickness. There should be little or no tension on the

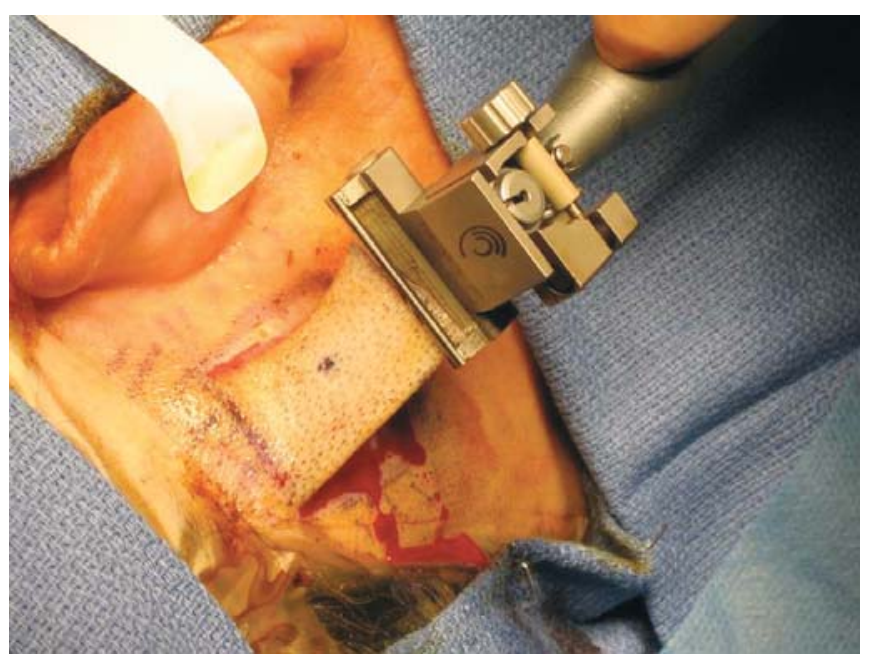

Fig. 6: Fast surgery flap elevation with a dermatome 
skin flap, when reapproximating the flap to the skin edges. Thinning the periosteum and creating a tension-free skin flap helps prevent movement of the skin flap during healing. A dressing is positioned over the abutment. It is held in place by the temporary white plastic healing cap, which snap-fits into the abutment. The head bandage is taken off the day after surgery. The plastic healing cap is left alone and removed at first follow-up visit, some two weeks following surgery. This will require healing for 3 to 6 months, at which point the BAHA can be fitted.

In a single stage or fast procedure, all the steps from creating a skin flap, reducing the soft tissue to drilling and insertion of fixture abutment and bringing it out through the skin flap (Fig. 7) is done at one time. The processor can be fitted (Fig. 8) three months after the surgery once the wound is healed and osseointegration has been completed.

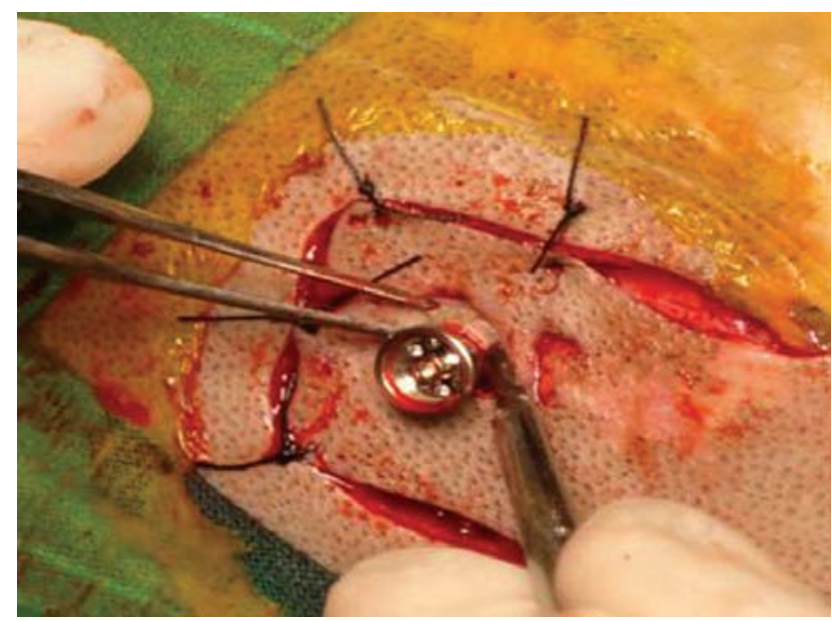

Fig. 7: Fast surgery easing the skin over the abutment

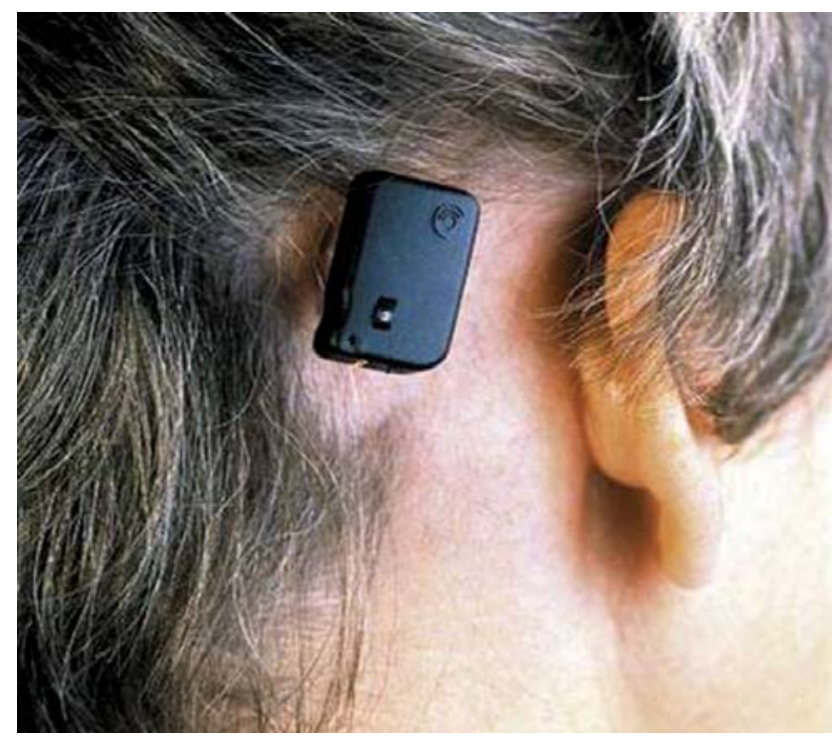

Fig. 8: Fitting of BAHA processor
Surgery to implant a BAHA fixture is not an ear operation since the ear itself is not operated on. Mastoid air cells decrease bone-titanium contact and should be avoided.

\section{Complications of BAHA Insertion}

1. Infection: Local wound inflammation around the abutment is commonly classified according to the clinical scoring system of Holgers and colleagues, ${ }^{9}$ as follows: Grade 0: No irritation;

Grade 1: Slight redness;

Grade 2: Red and moist;

Grade 3: Same as 2, but also with granulation tissue formed; and

Grade 4: Skin irritation of such a degree that the abutment has to be removed.

Treatment of the skin reaction may vary, depending on its grade. For Grade 1 reactions, local antibiotic ointment is recommended. Reapplying the healing cap, and wrapping the area with antibiotic gauze for a period of time may treat Grade 2 reactions. Grade 3 and 4 reactions require revision surgery.

Inflammation around the abutment is, in general, more common in children than in adults. ${ }^{10}$ Persistent, chronic infections around the implant may be due to Staphylococcus aureus. More severe infections can also occur, ranging from osteomyelitis with loss of the fixture, to intracerebral abscess. ${ }^{11}$

2. Failure of osseointegration: Symptoms and signs of osseointegration failure can vary. In the most severe situation, the abutment-fixture complex may be so loose that it falls out. At other times, a fibrous attachment is present; the fixture remains in place but the patient experiences little or no sound, or complains of sound distortion when the sound processor is fitted. In these situations, the abutment-fixture complex will spin freely when an attempt is made to tighten the abutment in the office. Several factors must be considered for adequate osseointegration. Proper surgical technique at the initial surgery is paramount. The thickness of the bone is also important. The thickness of the temporal bone is often a function of the patient's age at implantation and his/ her craniofacial anatomy. Patients with craniofacial syndromes often have thin bone in the area of planned implantation. One reason for implant losses without any known trauma is idiopathic bone resorption at the bonemetal interface. ${ }^{12}$

3. Bone overgrowth: The possibility of bone overgrowth should be considered when a loose abutment cannot be tightened. Bone overgrowth is found exclusively in children, especially between the ages of 5 and $11 .{ }^{13}$ Bone 
growth during this time period is significant, with the growth of the temporal and parietal bones being appositional (i.e. lamellar). Because growth is appositional, bone overgrowth can occur while the implant position does not change in the sagittal direction.

\section{MODELS}

Currently four BAHA systems are available:

- BAHA Cordelle II: It is a body worn BAHA for people with a severe hearing loss who need more amplification. It compensates up to $65 \mathrm{dBHL}$ of hearing loss. The Cordelle II consists of a transducer which snaps onto the abutment and a body worn unit. This is the only BAHA to have an induction telecoil receiver built-in.

- BAHA Divino: It is a digital BAHA with a built-in directional microphone and compensates for $45 \mathrm{~dB} \mathrm{HL}$ of sensorineural hearing loss. Even though the Divino takes a while to get used to for adolescents, in the long run it has been proven to be successful for most ages.

- BAHA Intenso: More power and clearer sound quality in all types of listening environments plus far less irritation. It compensates for up to $55 \mathrm{~dB} \mathrm{HL}$ of sensorineural hearing loss.

- BP 100: It is the largest generation of BAHA processors which is slimmer and smaller than the previous generation processors. It uses signal processing and fitting strategies uniquely developed to take advantage of bone conduction hearing.

\section{BAHA IN CHILDREN}

In the United States, the FDA requires that children be at least 5 years old to ensure adequate skull maturity before receiving implant surgery. Researchers at the Hospital for Sick Children in Toronto, Ontario have performed implants in children as young as 14 months of age and conclude that age does not appear to be an impediment for the successful use of bone-anchored aids. In their view, younger patients benefit from earlier speech and language acquisition, according to an online news article. ${ }^{14}$

For children in the United States or elsewhere deemed too young for implant surgery, a Soft band (Fig. 9) is available with the sound processor connected to a plastic snap connector sewn into the band and held against the skin through the pressure from the band. Researchers in the Netherlands concluded that the Soft band was a "valid intervention in children with congenital bilateral aural atresia who were too young for percutaneous BAHA application.”15 These children can then have a fixed BAHA once the skull thickness increases to more than $3 \mathrm{~mm}$.

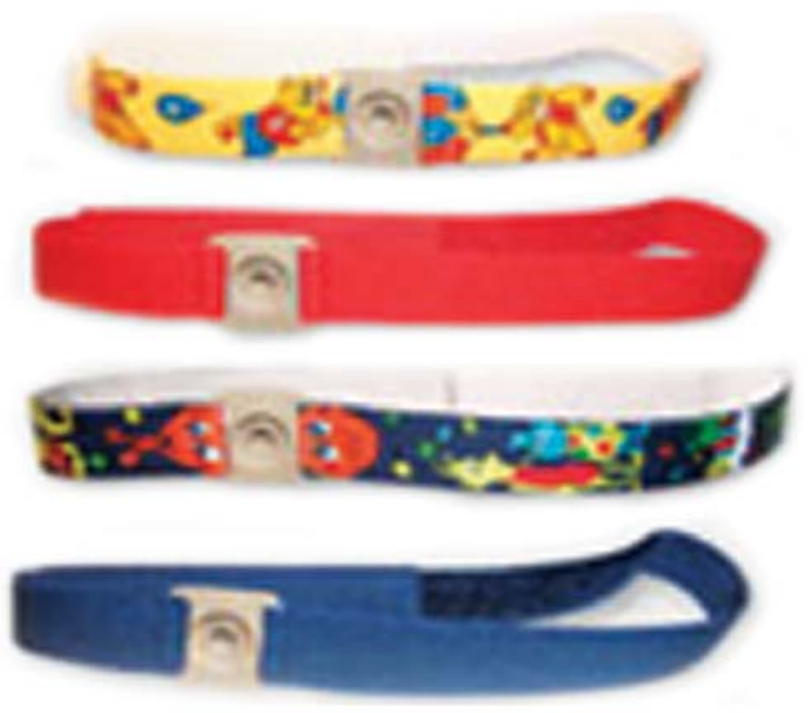

Fig. 9: Soft band (Courtesy: Cochlear Ltd., Australia)

\section{BAHA CARE}

1. The patient is advised to clean the area around the abutment daily: Washing hair will soften any crust. The patient is also advised to use the soft cleaning brush supplied with the BAHA kit and gently wipe the bristles against the side of the abutment, not the skin. All debris around or inside the abutment should be removed and use of antibacterial soap is recommended. Then the skin should be dried gently.

2. Patient should not allow hair to wrap itself around the abutment.

3. Patient should avoid blowing hot air from a hair dryer on the abutment for a long period.

4. It is advisable to cover the abutment and skin graft site with plastic to protect the skin and the abutment from the chemicals especially whenever strong chemicals, such as hair dying solutions, are being applied to your hair as the skin surrounding the abutment may become red, swollen, infected, or burned.

5. The external BAHA processor should be removed before undergoing MRI imaging.

6. For best benefit, it should be worn all through the day.

7. If the processor whistles when touched or when it comes into contact with other objects then the whistling can be reduced by simply repositioning the processor.

8. During windy conditions, outdoors, if the directional microphones may pick up wind sounds, the processor needs to be rotated on the abutment 90 degrees or until the wind sound stops. 


\section{CONCLUSION}

BAHA is ideal for those who have problems with their outer and middle ears and of course for those with unilateral deafness who cannot use conventional hearing aids. In addition, it is very comfortable to use surpassing all other types of hearing aid solutions, and it only requires to be implanted properly, after which the patient will not even notice its presence nor will others be aware that it is being worn. It also does not slip off or loosen unlike conventional hearing aids and also does not require any refitting once it has been successfully implanted.

\section{REFERENCES}

1. Tietze L, Papsin B. Utilization of bone-anchored hearing aids in children. Int J Pediatr Otorhinolaryngol 2001;58:75Y80.

2. Mylanus EAM, Cremers CWRJ. A one-stage surgical procedure for placement of percutaneous implants for the bone-anchored hearing aid. J Laryngol Otol 1994;108:1031Y5.

3. Mylanus EA, van der Pouw KC, Snik AF, Cremers CW. Intra individual comparison of the bone-anchored hearing aid and air conduction hearing aids. Archives of Otolaryngology-Head and Neck Surgery 1998;124:271-76.

4. Lustig LR, Arts HA, Brackmann DE, et al. Hearing rehabilitation using the BAHA bone-anchored hearing aid: results in 40 patients. Otology and Neurotology May 2001;22(3): 328-34.

5. Lin LM, Bowditch S, Anderson MJ, May B, Cox KM, Niparko K.Amplification in the rehabilitation of unilateral deafness; speech in noise and directional hearing effects with bone-anchored hearing and contralateral routing of signal amplification. Otology and Neurotology 2006;27(2):172-82.

6. Zeitoun H, De R, Thompson SD, et al. Osseointegrated implants in the management of childhood ear abnormalities: With particular emphasis on complications. J Laryngol Otol 2002;116(2): 87-91.

7. Granstrom G, Bergstrom K, Odersjo M, et al. Osseointegrated. implants in children: Experience from our first 100 patients. Otolaryngol Head Neck Surg 2001;125(1):85-92.

8. Tjellstro“ mA, Granstrom G. One-stage procedure to establish osseointegration: A zero to five years follow-up report. J Laryngol Otol 1995;109(7):593-98.

9. Holgers KM, Tjellstro“ MA, Bjursten LM, et al. Soft tissue reactions around percutaneous implants: A clinical study on skin-penetrating titanium implantsts used for bone-anchored auricular prostheses. Int J Oral Maxillofac Implants 1987;2(1): 35-39.

10. Papsin BC, Sirimanna TK, Albert DM, et al. Surgical experience with bone-anchored hearingaids in children. Laryngoscope 1997;107(6):801-06.

11. Deitmer T, Krassort M, Hartmann S. Two rare complications in patients with boneanchored hearing aids. Laryngorhinootologie 2003;82(3):162-65.

12. Reyes RA, Tjellstro“ MA, Granstrom G. Evaluation of implant losses and skin reactions around extraoral bone-anchored implants: A 0- to 8-year follow-up. Otolaryngol Head Neck Surg 2000;122(2):272-76.

13. Jacobsson M, Albrektsson T, Tjellstro“ MA. Tissue-integrated implants in children. Int JPediatr Otorhinolaryngol 1992; 24(3):235-43.

14. Bone-anchored hearing aids successfully implanted in very young children. Reuters Health Information. Accessed online at www.medscape.com/viewarticle/550794_print

15. Hol MK, Cremers CW, Coggens-Schellekens W, Snik AF. The BAHA softband. A new treatment for young children with bilateral congenital atresia. International Journal of Pediatric Otorhinolaryngology 2005;69(7):973-80. 